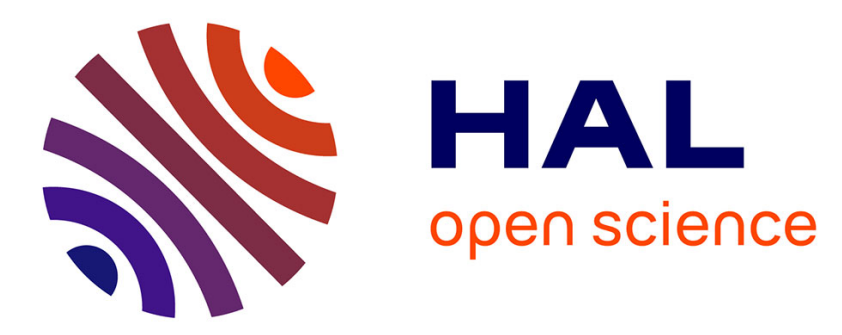

\title{
Interactive procedural simulation of paper tearing with sound
}

Thibault Lejemble, Amélie Fondevilla, Nicolas Durin, Thibault Blanc-Beyne, Camille Schreck, Pierre-Luc Manteaux, Paul G. Kry, Marie-Paule Cani

\section{- To cite this version:}

Thibault Lejemble, Amélie Fondevilla, Nicolas Durin, Thibault Blanc-Beyne, Camille Schreck, et al.. Interactive procedural simulation of paper tearing with sound. 8th ACM SIGGRAPH Conference on Motion in Games, MIG '15, Nov 2015, Paris, France. pp.143-149, 10.1145/2822013.2822029 . hal-01206764

\section{HAL Id: hal-01206764 \\ https://hal.inria.fr/hal-01206764}

Submitted on 3 Nov 2015

HAL is a multi-disciplinary open access archive for the deposit and dissemination of scientific research documents, whether they are published or not. The documents may come from teaching and research institutions in France or abroad, or from public or private research centers.
L'archive ouverte pluridisciplinaire HAL, est destinée au dépôt et à la diffusion de documents scientifiques de niveau recherche, publiés ou non, émanant des établissements d'enseignement et de recherche français ou étrangers, des laboratoires publics ou privés. 


\section{Interactive procedural simulation of paper tearing with sound}

\begin{abstract}
Thibault Lejemble ${ }^{1}$
Camille Schreck ${ }^{2}$

${ }^{1}$ ENSIMAG Grenoble INP *

\author{
Amélie Fondevilla ${ }^{1}$
}

Pierre-Luc Manteaux ${ }^{2}$

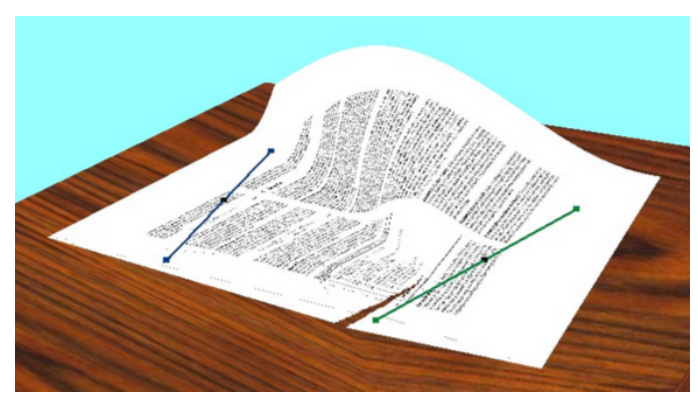
a multi-touch tablet.

\section{Abstract}

We present a phenomenological model for the real-time simulation of paper tearing and sound. The model uses as input rotations of the hand along with the index and thumb of left and right hands to drive the position and orientation of two regions of a sheet of paper. The motion of the hands produces a cone shaped deformation of the paper and guides the formation and growth of the tear. We create a model for the direction of the tear based on empirical observation, and add detail to the tear with a directed noise model. Furthermore, we present a procedural sound synthesis method to produce tearing sounds during interaction. We show a variety of paper tearing examples and discuss applications and limitations.
\end{abstract}

${ }^{2}$ University Grenoble-Alpes, CNRS (LJK), Inria ${ }^{\dagger}$
Thibault Blanc-Beyne ${ }^{1}$

Marie-Paule Cani ${ }^{2}$
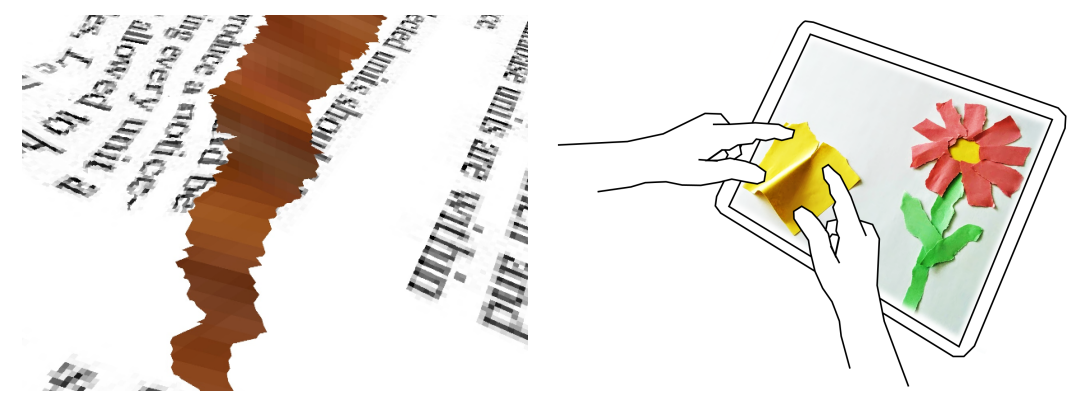

Figure 1: From left to right, an example result of our method showing detail added to the torn edge of the paper, a closer view of the geometry of the tear, and an illustration of an example application of our technique using interactive tearing paper for torn paper collage creation on

CR Categories: I.3.7 [Computer Graphics]: Three-Dimensional Graphics and Realism-Animation;

Keywords: tearing, paper, sound, real-time

\section{Introduction}

Although it is a common material, paper is seldom represented in virtual environments. Indeed, paper has complex behaviors that are challenging to model and simulate, such as bending, crumpling, cutting, and tearing, while having resistance to shearing and stretching. There is a collection of successful techniques in recent research that can produce these behaviors through physics-based simulation methods, but not in real-time. In this paper we present the first tech-

\footnotetext{
*thibault.lejemble@ensimag.grenoble-inp.fr

$\dagger\{$ camille.shreck|pierre-luc.manteaux $\mid$ marie-paule.cani $\} @$ inria.fr

${ }^{\ddagger}$ kry@cs.mcgill.ca
}

nique in computer graphics for real-time paper tearing with a fast phenomenological model for both geometry and sound.

Our goal is an interactive method that allows easy manipulation and tearing of virtual paper for games or artistic applications, such as the virtual crafts application proposed in Figure 1. Thus, in addition to the realism of the results, we must ensure real-time computation. Physical simulations are not well suited because the high rigidity required to accurately simulate paper often causes numerical instabilities. Additionally, the number of degrees of freedom necessary to obtain a sufficient level of detail leads to prohibitive computational costs. That is why we choose to use a phenomenological approach to procedurally reproduce the behavior of paper being torn.

For interaction, we focus on the simplified case shown in Figure 1 where the movement of each hand is defined by the index and thumb and stays in a 2D plane; although the sheet of paper is initially flat, rotation of the hands produces tearing and 3D deformations due to out of plane buckling.

Paper is composed of microscopic fibers which have an important influence on the behavior of paper at a macroscopic level. To reproduce the behavior, we identify and focus on three features in our procedural model: isometric deformations, stochastic tearing, and the spectral energy of tearing sounds. Isometry of paper deformation comes from the fact that the fibers are oriented in all directions and cannot stretch or compress. Thus, upon any deformation, the surface of the paper preserves distances according to its initial flat state (i.e., the surface of the paper is developable). The stochastic nature of the shape of a tear can similarly be attributed to the random distribution of fibers. While the main direction of tearing is imposed by the movement of the hands, details along the tear exhibit variation due to tear-growth favoring the direction of fibers at the crack tip. Finally, to have realistic virtual paper, it is important to also provide sound that matches the animation. The sound of paper tearing is caused by the breaking of fibers, and we note the distribution of fibers and speed of the tear has an important effect on the sound spectrum and the creation of a plausible auditory signal.

Our contributions are a real-time solution for the challenges of modeling and animating detailed tear shapes and paper buckling, and a 
model for generating plausible tearing sounds. The rest of this article is organized as follows. We first summarize the related work. Then we explain the method: the isometry-preserving model of deformation, the algorithm to find the path of the tear, and the model for the sound. Finally, we evaluate the results and discuss limitations and future work.

\section{Related work}

Modeling deformation and sound is a common objective in computer animation research with interactive applications such as games. In the context of tearing paper, we can divide the related work on deformation and tearing into several categories: physically based methods, geometric methods, and hybrid methods that mix physics and geometric approaches. Equivalently, related work on interactive sound synthesis tends to be either physics- or examplebased, or more commonly, a combination of both.

\subsection{Deformation and tearing}

The simulation of paper is similar to that of cloth, which is a topic that has been investigated extensively in computer graphics over the last three decades. In recent research, inextensible cloth models [Goldenthal et al. 2007] and strain limiting techniques [Thomaszewski et al. 2009; Wang et al. 2010] have helped increase the quality of cloth simulations. While these techniques do not address the isometry of paper deformations, it is possible to explicitly take into account the developable nature of the surface through adaptive meshing, thus improving the simulation of physically based paper crumpling [Schreck et al. 2015].

In computer animation, tearing of cloth and thin shells has received less attention in comparison to the fracture of solids. Metaaphanon et al. [2009] present a method for simulating the tearing of cloth that also produces fraying at the edges. Although it makes sense to break cloth using the structure of fibers, using lower resolution cloth simulations and discrete cuts along existing edges allows for interactive simulation with lower fidelity results [Souza et al. 2014]. In general, these methods do not transfer well to the randomly distributed fibers in paper, where treating the surface as a continuum and remeshing to accommodate fracture is preferable. The method of Busaryev et al. [2013] adapts the mesh to the fracture, and furthermore presents high-quality off-line simulations of tearing paper that include multiple layers. Including plasticity in the simulation allows for crumpling [Narain et al. 2013], and more recently, Pfaff et al. [2014] present adaptive meshing with both plastic deformation and fracture, permitting simulations of crumpling and tearing.

Physically based simulations produce excellent results, but not without large computational cost. In contrast, our approach to producing interactive deformations is a purely geometric approach and is therefore closer in spirit to kinematic methods. For instance, Sumner et al. [2005] demonstrate pose changes for a cloth sheet using an example-based inverse kinematics method that exploits as-rigid-as-possible mesh deformation. Other work on geometric methods directly addresses developability in flexible surfaces. Rohmer et al. [2011] use fold lines to direct procedurally generated static geometry of folded paper that approximately respects isometry. In contrast, Solomon et al. [2012] present an interactive developable surface modeling system that enforces exact developability.

\subsection{Interactive sound synthesis}

Early examples of sound simulation in computer animation use physics simulation and phenomenological impact and roughness models to drive modal vibration models [van den Doel et al. 2001].
Modal vibration frequencies and decays can be estimated from data [Pai et al. 2001], or computed using modal analysis of finite element models [O'Brien et al. 2002; Picard et al. 2010]. Accounting for acoustic transfer significantly increases the quality of modal sounds [James et al. 2006], and similarly, propagation and scattering are important in larger scale virtual environments [Manocha and Lin 2009]; however, we do not address these effects in our work. Furthermore, we note that tearing sounds are largely due to the breaking of fibers, and the resulting sounds are not well modeled by physically based modal vibrations.

In computer animation, the fracture of brittle elastic objects has been investigated with fast physically based sound synthesis methods that make use of simplified fragment vibration models and acceleration noise [Zheng and James 2010; Chadwick et al. 2012]. But tearing is quite different because the object being torn divides slowly into two parts, while methods for fracture sounds typically assume that the separation of fragments occurs at a much smaller time scale.

Data-driven techniques include methods that break up recorded sounds into small chunks, and recombine them to make new sounds [Schwarz 2006]. Such methods can be described as concatenative techniques, and they have been useful in the production of clothing sounds [An et al. 2012]. Example based techniques could be very useful in synthesizing paper tearing sounds, but ultimately we note that the much simpler approach of matching the noise spectrum of examples provides compelling and plausible results. We note that the acoustic emissions from fractures of paper and their characteristic power-law statistics has also been studied by the physics community [Salminen et al. 2002].

\section{Paper deformation model}

As mentioned above, simulating the behavior of a paper sheet under tearing remains a challenge for interactive computer graphics applications. Instead of using a mechanical simulation to reproduce this behavior, we choose to create a phenomenological model based on observations. Our model is able to reproduce plausible paper tearing in real-time, thus offering the possibility of interaction.

In order to capture the key features of paper deformation, we performed several informal experiments and observed the behavior of tearing paper. The setting of our experiments (and our model) is a paper sheet placed on a planar surface and manipulated with two hands. The thumb and index finger of each hand is in contact with the paper, and together they apply a rotation that deforms and tears the paper. An example observation can be seen in Figure 2a.

We observe that paper is inextensible, and note that this implies that it is developable, i.e., it can be unfolded onto a plane without being stretched or compressed. Therefore, as soon as paper begins to tear, it must also bend in order to preserve the distances. We also observe that paper stays smooth, and we assume that we can model the surface as a $C^{2}$ developable surface consisting of a set of parts: planes, cylinders, cones, and tangent developables. As the point at crack tip represents the one and only singular point of the surface in our model, we chose to define the bending portion of the paper as a generalized cone. As shown in Figure 2b, we thus distinguish three specific regions in our model: two planar regions, and a generalized cone. The cone is delimited by the two lines formed by the crack tip and each thumb, and defined by the set of lines through the apex $s$ at the crack tip and the points of a $C^{2}$ curve, as seen in Figure $2 \mathrm{c}$.

In our model, each hand is modeled by two points that represent the index finger and the thumb. They can be placed anywhere on the paper sheet. At each time step, the hands perform a rotation, which we denote $\Delta \theta_{1}$ for the left hand and $\Delta \theta_{2}$ for the right. This hand 


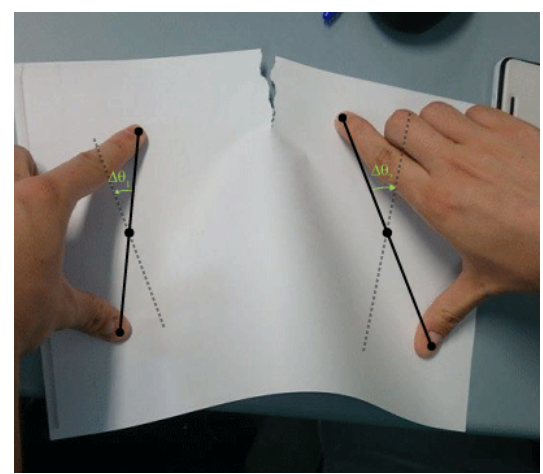

(a)

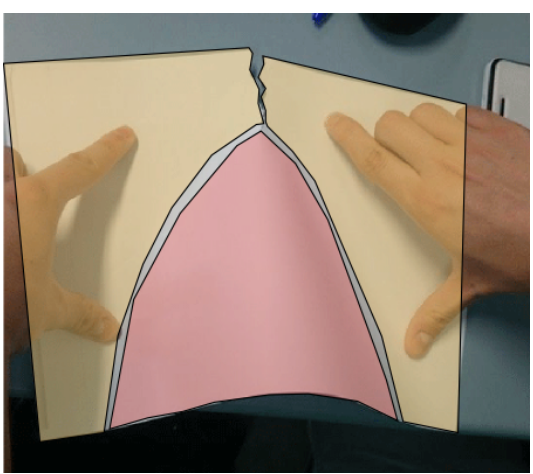

(b)

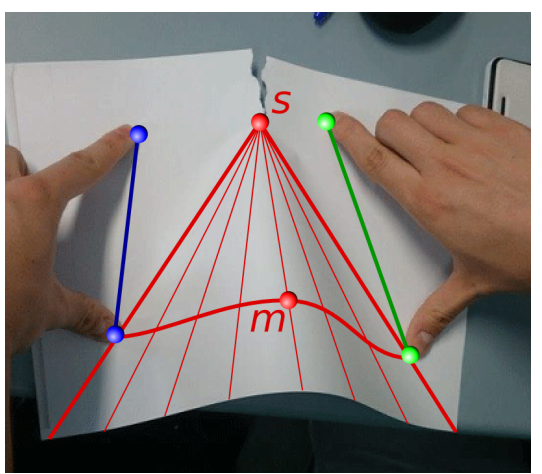

(c)

Figure 2: In (a), the model for the movement of the hands. In (b), the two planar regions in yellow and the generalized cone in red. In (c), an example of the cone shape formed between the tip of the tear s and the thumbs. The position and orientation of the two flat portions of the paper on either side of the cone are driven by the motion of the hands. The cone is defined by its apex s and two Hermit curves joining, respectively, each thumb and the vertex $m$ at the middle of the geodesic passing though both thumbs.

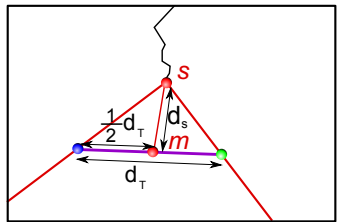

(a) 2D configuration

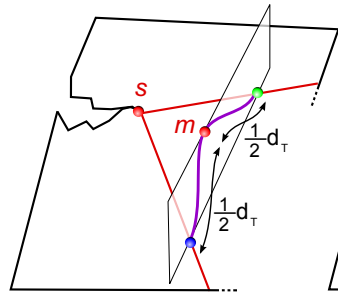

(b) 3D bend

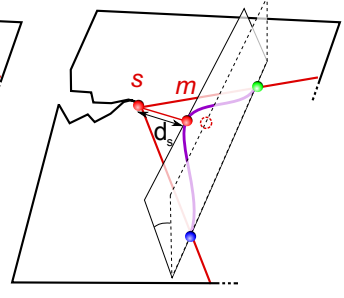

(c) 3D tilt for isometry

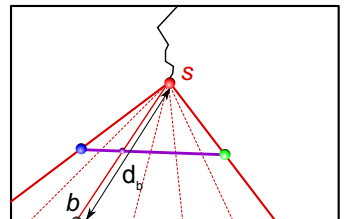

(d) 2D triangulation

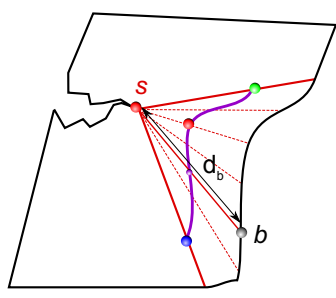

(e) $3 \mathrm{D}$ triangulation

Figure 3: Construction of the generalized cone begins $(a)$ with the $2 D$ position of $m$ set at the midpoint between the thumbs, and (b) with the $3 D$ position of $m$ initially set vertically above the plane such that the Hermite curves preserve the distance between the thumbs. The $3 D$ position of $m$ is then rotated about an axis going through the thumbs $(c)$ to ensure the distance between $m$ and the apex $s$ matches the distance in the initial flat state. Finally, the cone is rasterized $(d)$ in the initial flat state, and $(e)$ the $3 D$ positions of the points on the border of the paper are computed such that they on the corresponding line and the distance between border points and the cone apex is preserved.

motion induces tearing (as described in Section 4) and a deformation of the surface between the thumbs. The planar regions follow a rigid motion directly imposed by the hands, rotating around the crack tip at each step by the corresponding angle of hand rotation.

The process of building the generalized cone is shown in Figure 3. We approximate the geodesic curve that joins the two thumbs by two Hermite cubic splines which meet at vertex $m$, at an equal distance to each thumb. The constraints to compute the Hermit curves are the position of each thumb, the point at the top of the bend $m$, and the tangents at those vertices. We choose all tangents to be parallel to the line between the thumbs to simulate the pressure applied by the thumbs on the paper. We select in advance an appropriate magnitude for the tangent to correspond to the size and type of paper. The vertical position of the middle point $m$ is computed using an iterative algorithm such that the length of the curve between $m$ and each thumb matches the distance between them in the initial 2D configuration. In order to preserve the distance between crack tip $s$ and the points of the curve, we rotate the curve such that the distance between $m$ and $s$ on the 3D shape corresponds to the one on the 2D pattern. The generalized cone is then defined by the apex $s$ and the curve between the thumbs. To find each vertex $b$ on the curved border of the paper, we compute points that preserve the distance between $s$ and $b$ along the corresponding line.

\section{Tearing model}

Paper tearing in real-time is a challenging problem. In physicsbased simulation, the crack direction at each time step is based on the eigenvalues of the stress tensor at the crack tip, and is perpendicular to the greatest stress direction. In the case of simulated paper, an extremely fine discretization is necessary to produce realistic small scale details of torn paper. This makes stress-based crack direction computation inappropriate for real-time paper tearing.

Instead, we build a procedural tearing algorithm that exhibits the characteristic stochastic details of torn paper that we observe in empirical experiments. Our algorithm is based on two observations. First, the main crack direction is guided by the motion of the hands. Second, the details along the crack are due to the distribution of fibers and a resistance to stress that is not uniform across the paper's surface. Our algorithm is therefore made of two steps. We first compute a main tearing direction based on hand motion, and then add tearing details based on an anisotropic fiber distribution model.

\subsection{Tearing direction}

We observe that tearing initiates at a random position on the edge between the two index fingers. In our model, we therefore choose to initiate tearing at a random point along the edge of the sheet, specifically, at a point between where the rays from thumb to index positions intersect the edge. Then, at each time step $i$, we compute the position of the next crack tip $s_{i}$ according to the motion of the hands. For this, we define a vector $\vec{t}$ that represents the advance of the tear between $s_{i}$ and the previous crack tip position $s_{i-1}$, as shown in Figure 4. 


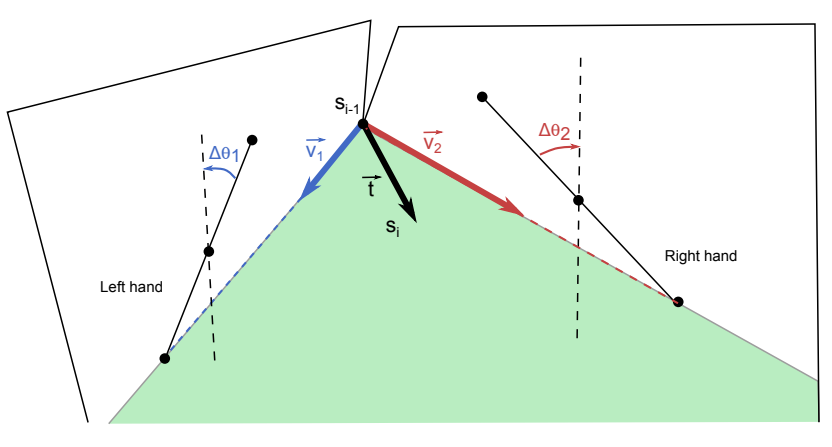

Figure 4: Our model for determining the direction of the tear at each time step depends on the incremental rotation of each hand.

In our experiments, we observe that rotating a single hand produces a tear in the direction of the thumb of that hand. Therefore we compute $\vec{t}$ as a linear combination of the vectors $\vec{v}_{1}$ and $\vec{v}_{2}$ defined by the crack tip $s$ and thumb of each hand. We weight each vector by the corresponding rotation angle $\Delta \theta$ to compute the current tearing direction

$$
\vec{t}=C\left(\Delta \theta_{1} \frac{\vec{v}_{1}}{\left\|\vec{v}_{1}\right\|}+\Delta \theta_{2} \frac{\vec{v}_{2}}{\left\|\vec{v}_{2}\right\|}\right),
$$

where $C$ is a constant that controls the tearing speed. That is, the norm $\|\vec{t}\|$ is the speed of the tear, and it is used to parametrize the sound as described in Section 5.

\subsection{Tearing details}

Advancing the tear using only the tear direction computed above will not produce the rough edges we see in real torn paper. Therefore we procedurally add fine details along the path between two successive crack tip positions. Our algorithm is inspired by the work of Chen et al. [2014], and makes use of two 2D functions as shown in Figure 5. A Perlin noise function $P$ lets us represent the stress resistance of fibers at each point of the paper, while a Gaussian function models the stress field $G_{i}$ produced by hand motion between the steps $i-1$ and $i$. Specifically, the Gaussian is centered on the edge between $s_{i-1}$ and $s_{i}$. We combine these two functions to obtain a $2 \mathrm{D}$ texture

$$
\nu_{i}(x, y)=(1-p) P(x, y)+p / G_{i}(x, y),
$$

where each texel can be considered as a breaking coefficient that intuitively represents the probability that the tear will go through this texel. The parameter $p$ lets us adjust the blend between the two functions to achieve a desired rough appearance of the tear.

From this texture, the tear detail can be chosen as the shortest path, but we prefer a more intuitive and simple approach. Going from the texel representing $s_{i-1}$, we iteratively choose the neighboring texel with the greatest breaking coefficient until we reach the destination $s_{i}$. To ensure that we actually reach $s_{i}$ without going backward, we only consider the texels in directions that form an angle less than $\frac{\pi}{2}$ with the vector between the current texel and the destination $s_{i}$.

\section{Sound}

Realistic auditory feedback plays an important part in the creation of compelling virtual experiences. We enhance our interactive animation by synthesizing plausible sounds for the virtual paper as it is torn. We choose to create the sound with a very simple and fast procedural algorithm that has minimal computational and memory costs. Our method is based on observations made from audio
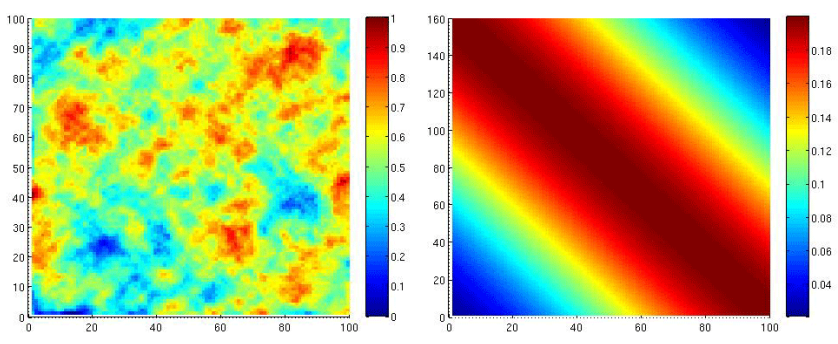

Figure 5: Variation in the tearing direction is produced with the combination of $2 D$ Perlin noise (left) with a $2 D$ Gaussian function (right) aligned with the current tear-trajectory direction.

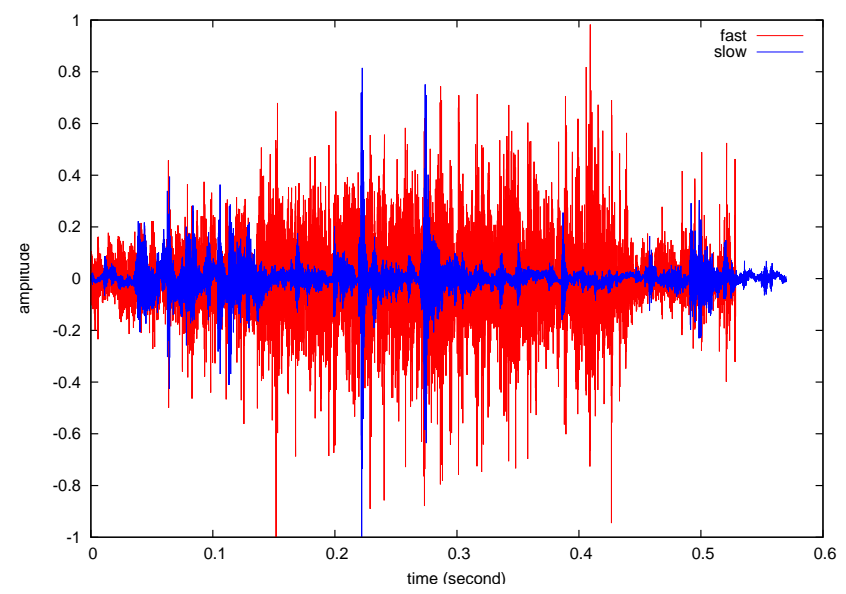

Figure 6: Comparison between the audio signal of a fast tear (red) and a slow tear (blue). The amplitude of the fast tearing is larger than the slow one, while the large peaks are generally closer for the fast tear.

recordings of real paper, and hypotheses concerning the breaking of fibers and the speed of the tear.

The sound of tearing paper is caused by fibers or links between them being broken. With the random distribution of fibers in the paper, this process is largely stochastic, and therefore sounds like noise (see Figure 7). Indeed, white noise makes a good starting point for tearing sound synthesis given the largely flat nature of the spectral power density of the sounds we recorded. Each fiber produces an energy burst when being torn apart. As previously noted, the fiber distribution is anisotropic across the paper surface. This produces sudden changes of energy in the sound and resembles crackling, which is visible in the slow tearing example (blue) shown in Figure 6. We reproduce the phenomenon by first generating a white noise and then multiplying it by $r^{\alpha}$, where $r$ is a random number between 0 and 1 , and the exponent $\alpha$ is used to tune the sound to be something between white noise and a crackling noise. When $\alpha=1$ the sound is a white noise, while for a greater value of $\alpha$ the sound will contain clearer peaks of energy. In our examples, we use $\alpha=100$.

The crack propagation speed also affects the sound. First, the sound energy changes according to the speed, as can be seen the comparison between a slow tearing and a fast tearing in Figure 6. This can be explained by the fact that a faster tear involves a larger number of broken fibers in the same amount of time. Second, the amplitude changes that are caused by varying density of fibers across the sheet will also occur faster. Thus, we linearly tune the amplitude and pitch of the modified white noise according to the speed. 


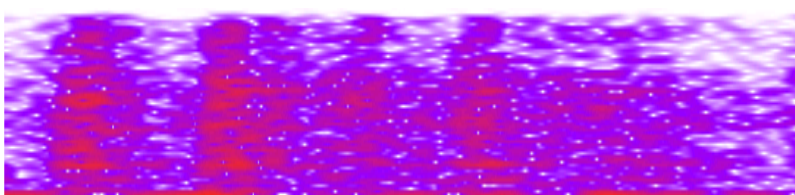

(a) Slow tearing

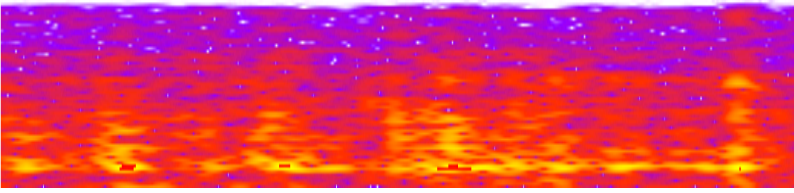

(b) Fast tearing

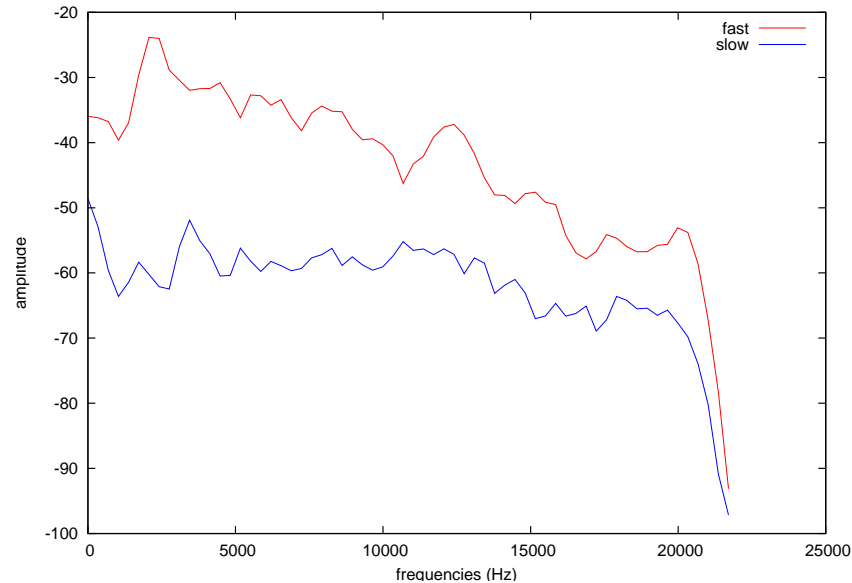

equencies $(\mathrm{Hz})$

Figure 7: Spectrograms of 0.05 s clips from the middle of the recorded sounds shown in Figure 6, with a plot of the spectral power densities. All frequencies are present at a relatively constant speed-dependent power, with a small frequency dependent power drop for fast tearing.

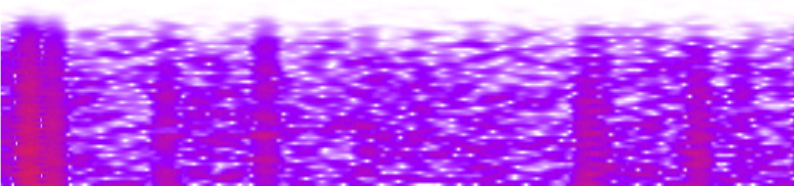

(a) Slow tearing

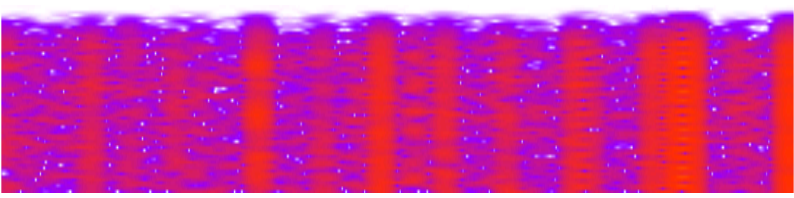

(b) Fast tearing

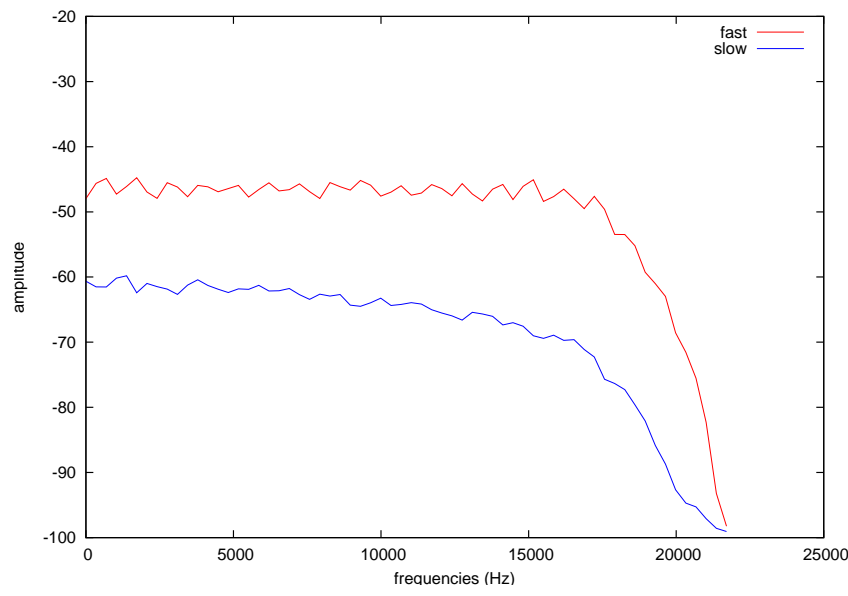

Figure 8: Spectrograms of 0.05 s clips of synthesized sounds, with a plot of the spectral power densities.
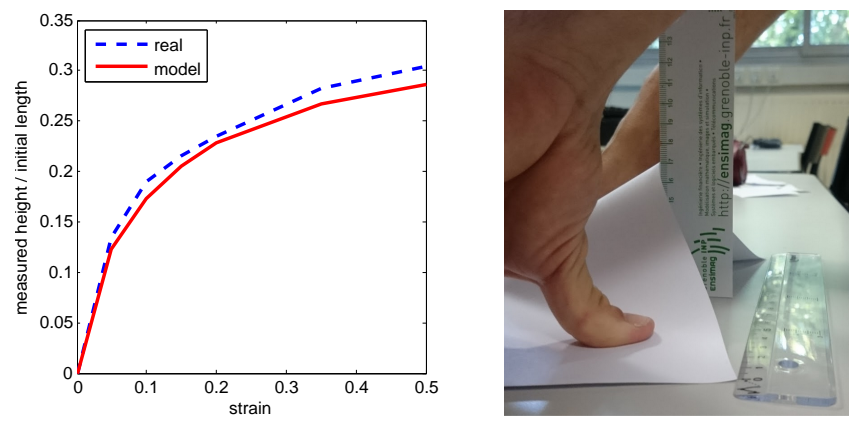

Figure 9: Experimental evaluation shows that our cone deformation model produces results that correspond to real paper.

\section{Results and discussion}

Our system is implemented in $\mathrm{C}++$, using OpenGL for graphics and the FMOD library for sound synthesis. We use scripted motions to produce different real-time simulations of tearing paper with sound. An example of our results along with a close-up image showing de- tail along the tear can be seen in Figure 1, but please see the accompanying video for additional examples that also include synthesized tearing sounds.

We performed a simple experimental evaluation of our deformation model. Figure 9 shows a comparison of the cone height produced by our model with that of a real sheet of paper. Specifically, we compare the real and simulated cone height normalized by original distance between the thumbs at different amounts of strain (i.e., compression, normalized by the original distance between the thumbs). The results suggest that our selection of the magnitude of Hermite curve tangents in our model works well for the scale of paper and typical interactions (e.g., thumb placement as shown in the figure). We note that our model could be improved by making the tangent magnitude a function of the type of paper and the original distance between the thumbs.

In Figure 8, we show spectrograms and spectral power density plots of synthesized slow and fast tearing sounds. We are able to capture the noise and crackling features of tearing, but in comparison to recorded sounds, our synthesized sounds focus only on the tear and are therefore missing several important qualities related to the cone. Specifically, the cone acts as a resonator and its size and height influence the sounds created in real tearing. Further research is nec- 
essary to identify how the cone parameters can be included in our procedural sound synthesis method.

\section{Conclusions and Future Work}

In this paper we present a first geometric model for paper that can be torn in real time with integrated sound. The approach has several limitations that we would like to address in future work. Notably, the paper primarily lies in a plane (e.g., a virtual table top). While parts of the paper can leave the surface of the table during interactions, the placement and motions of the hands that drive the deformation and tearing of paper are also confined to the plane. We note that this constraint on interaction is not necessarily an important limitation in the context of how we envision our method used on multi-touch tablets or tables (e.g., Microsoft PixelSense). More importantly, in our current method the hands can only rotate about the crack tip, while translation must be addressed to have a general solution for table-top tearing. Furthermore, we only track the frontier of one tear, while many paper tearing scenarios involve the creation of multiple simultaneous tears and branching [Manteaux et al. 2015]. We also note that we could take into account the weight of the paper, and how this influences the tear and the sound. Finally, we note that multiple layers of fibers tend to give a rough edge to torn paper and we leave the addition of these details to future work.

\section{Acknowledgements}

This work was partially funded by the advanced grant no. 291184 EXPRESSIVE from the European Research Council (ERC-2011ADG_20110209).

\section{References}

An, S. S., James, D. L., And Marschner, S. 2012. Motiondriven concatenative synthesis of cloth sounds. ACM Trans. Graph. 31, 4 (July), 102:1-102:10.

BusAryeV, O., Dey, T. K., AND WANG, H. 2013. Adaptive fracture simulation of multi-layered thin plates. ACM Trans. Graph. 32, 4 (July), 52:1-52:6.

Chadwick, J. N., Zheng, C., And JAmes, D. L. 2012. Faster acceleration noise for multibody animations using precomputed soundbanks. In Proceedings of the ACM SIGGRAPH/Eurographics Symposium on Computer Animation, SCA '12, 265-273.

Chen, Z., Yao, M., Feng, R., And Wang, H. 2014. Physicsinspired adaptive fracture refinement. ACM Trans. Graph. 33, 4 (July), 113:1-113:7.

Goldenthal, R., Harmon, D., Fattal, R., Bercovier, M., AND GRINSPUN, E. 2007. Efficient simulation of inextensible cloth. ACM Trans. Graph. 26, 3 (July).

JAMES, D. L., BARBiČ, J., AND PAI, D. K. 2006. Precomputed acoustic transfer: Output-sensitive, accurate sound generation for geometrically complex vibration sources. ACM Trans. Graph. 25, 3 (July), 987-995.

MANOCHA, D., AND LIN, M. 2009. Interactive sound rendering. In Computer-Aided Design and Computer Graphics, 19-26.

Manteaux, P.-L., Sun, W.-L., Faure, F., Cani, M.-P., And O'BRIEN, J. F. 2015. Interactive detailed cutting of thin sheets. In Proceedings of the Eigth International Conference on Motion in Games, ACM, New York, NY, USA, MIG '15.
Metaaphanon, N., Bando, Y., Chen, B.-Y., And Nishita, T. 2009. Simulation of tearing cloth with frayed edges. Computer Graphics Forum 28, 7, 1837-1844.

NArain, R., Pfaff, T., And O’Brien, J. F. 2013. Folding and crumpling adaptive sheets. ACM Trans. Graph. (Proc. of SIGGRAPH) 32, 4 .

O'Brien, J. F., Shen, C., And Gatchalian, C. M. 2002. Synthesizing sounds from rigid-body simulations. In Proceedings of the 2002 ACM SIGGRAPH/Eurographics Symposium on Computer Animation, SCA '02, 175-181.

Pai, D. K., Doel, K. V. D., James, D. L., LANG, J., Lloyd, J. E., Richmond, J. L., AND YAU, S. H. 2001. Scanning physical interaction behavior of $3 \mathrm{~d}$ objects. In Proceedings of the 28th Annual Conference on Computer Graphics and Interactive Techniques, SIGGRAPH '01, 87-96.

Pfaff, T., Narain, R., de Joya, J. M., And O'Brien, J. F. 2014. Adaptive tearing and cracking of thin sheets. ACM Trans. Graph. 33, 4 (July), 110:1-110:9.

Picard, C., Frisson, C., Faure, F., Drettakis, G., And KRY, P. 2010. Advances in modal analysis using a robust and multiscale method. EURASIP Journal on Advances in Signal Processing 2010, 1, 392782.

Rohmer, D., CANi, M.-P., Hahmann, S., AND Boris ThibERT. 2011. Folded paper geometry from 2D pattern and 3D contour. Eurographics Short Paper, 21-24.

Salminen, L., Tolvanen, A., And Alava, M. 2002. Acoustic emission from paper fracture. Physical Review Letters 89, 18 185503 .

Schreck, C., Rohmer, D., Hahmann, S., Cani, M.-P., Jin, S., WANG, C. C., AND BlOCH, J.-F. 2015. Non-smooth developable geometry for interactively animating paper crumpling. ACM Trans. Graph.. to appear.

SCHWARZ, D. 2006. Concatenative sound synthesis: The early years. Journal of New Music Research 35, 1, 3-22.

Solomon, J., Vouga, E., WARdetzky, M., And Grinspun, E. 2012. Flexible developable surfaces. Computer Graphics Forum (CGF), Proc. of Symposium on Geometry Processing (SGP) 31,5 .

Souza, M. S., Wangenheim, A., And Comunello, E. 2014. Fast simulation of cloth tearing. SBC Journal on Interactive Systems 5, 1, 44-48.

Sumner, R. W., Zwicker, M., Gotsman, C., ANd Popović, J. 2005. Mesh-based inverse kinematics. ACM Trans. Graph. 24, 3 (July), 488-495.

Thomaszewski, B., Pabst, S., and Strasser, W. 2009. Continuum-based strain limiting. Comput. Graph. Forum 28, 2, 569-576.

VAN DEN DoEl, K., KRY, P. G., AND PAI, D. K. 2001. Foleyautomatic: Physically-based sound effects for interactive simulation and animation. In Proceedings of the 28th Annual Conference on Computer Graphics and Interactive Techniques, SIGGRAPH '01, 537-544.

Wang, H., O'Brien, J. F., And Ramamoorthi, R. 2010. Multi-resolution isotropic strain limiting. ACM Trans. Graph. (Proc. of SIGGRAPH Asia) 29, 6, 160. 
ZHENG, C., AND JAMES, D. L. 2010. Rigid-body fracture sound with precomputed soundbanks. ACM Trans. Graph. 29, 4 (July), 69:1-69:13. 